\title{
La responsabilidad social corporativa en los principales grupos de comunicación españoles: incorporación, gestión y análisis de la información a través de sus páginas web
}

\author{
Recibido: 09 de diciembre de 2011 \\ Aceptado: 20 de junio de 2012 \\ Publicado: 31 de octubre de 2012
}

\author{
Jessica Fernández Vázquez \\ jessicafdez@uvigo.es \\ Universidad de Vigo (España)
}

\begin{abstract}
Resumen: La responsabilidad social corporativa (RSC) se ha convertido en un activo intangible e imprescindible en la gestión empresarial. Las instituciones son conscientes de que se integran en una comunidad con la que deben mantener un diálogo constante y en la que revierten parte de sus beneficios. De esta forma, la RSC se convierte en una herramienta clave de relaciones públicas a la hora de mejorar su reputación y legitimación social así como para transmitir una identidad corporativa determinada. En el caso de los medios de comunicación, la RSC cobra una doble dimensión: no sólo se trata de aplicar medidas responsables en torno al medio ambiente, grupos de interés, instituciones, etc. sino que debe transmitir la información de forma rigurosa, veraz, objetiva y ética. Teniendo en cuenta esto, decidimos estudiar la RSC de los principales grupos de comunicación en España a partir de la información publicada en sus páginas web.
\end{abstract}

Palabras clave: Responsabilidad social corporativa, grupos de comunicación, patrocinio, mecenazgo, relaciones públicas.

Abstract: Since the nineties Corporate Social Responsibility (CRS) has become one of the major assets in enterprise management. Businesses grew aware that their activities have strong consequences in the public sphere within a continuous dialogue between both of them, reverting part of the benefits in society. Hence, CSR is a key factor in terms of public relations to improve reputation and social legitimacy, not to mention its role within the corporate identity management. In case of media involved, CSR shows two approaches: on the one hand, implementing responsible measures as for environment, stakeholders, institutions, etc. and, on the other hand, facilitating information in a rigorous, true, objective 
and ethical way. This paper aims at studying CSR of the major communication groups in Spain through their web pages in the context of media as public opinion generators.

Key words: Corporate Social Responsibility, Communication Groups, Sponsorship, Patronage, Public Relations.

\section{Introducción}

Como indica Manfredi (2006: 376),

"la responsabilidad social consiste en el conjunto de obligaciones de las empresas hacia la sociedad en su proyecto empresarial. Incluye, aunque no está limitado, a los trabajadores, los consumidores de televisión (la audiencia), las organizaciones sociales (ONG, partidos políticos, sindicatos), alianzas institucionales y empresariales, anunciantes y socios tecnológicos. La RSC está vinculada a los principios del desarrollo sostenible, que defiende que las firmas están obligadas a tomar decisiones sobre el análisis económico financiero considerando las consecuencias sociales y medio ambientales de sus actividades".

En Europa y especialmente en España, la Responsabilidad Social Corporativa (RSC) o Empresarial (RSE), ha pasado de ser una gran desconocida a ser un elemento clave en las instituciones. La coyuntura económica y social vivida en la década de los noventa trajo consigo un cambio de mentalidad en las organizaciones, que comenzaron a ser conscientes de la importancia de la gestión de los activos intangibles para legitimarse a nivel social y crear valor más allá de los resultados económicos y financieros.

A partir de entonces, la RSC fue integrada de forma voluntaria en la gestión y planificación empresarial, pasando a tener un papel fundamental a la hora de transmitir una determinada identidad y comunicarse con los grupos de interés o stakeholders (Freeman, 1984). Esta necesidad de comunicación hace que la RSC se integre dentro de las Relaciones Públicas, cobrando especial importancia a la hora de hablar de legitimación, reputación y mejora de la imagen de las empresas. "Invertir en empresas con una política de RSE conlleva a largo plazo un aumento de valor para la empresa y en consecuencia para el accionista" porque, en términos generales y según el informe de Morgan Stanley y Oekmon Research (2004) estas empresas están mejor gestionadas a nivel general (Albareda y Balaguer, 2007: 21-22). Pero la RSC no se debe confundir, por lo tanto, "con acciones de patrocinio, mecenazgo, donaciones puntuales o estrategias de una corporación tendentes a un lavado puntual de imagen o de mejora de su reputación corporativa" (Fernández, 2011), porque existe interés propio además de compromiso social.

Teniendo en cuenta la importancia alcanzada por este fenómeno y valorando el papel social de los medios de comunicación como generadores de opinión pública y transmisores de la realidad, decidimos estudiar la RSC de los principales grupos de comunicación en España a partir de la información publicada en sus páginas web, ya que Internet se ha convertido en una herramienta clave para difundir información de las propias empresas. La muestra de 
estudio se compone de los sites de PRISA, Planeta, Gestevisión-Telecinco, Vocento, Godó, Intereconomía y Unidad Editorial.

\section{Objetivos, metodología empleada e hipótesis de partida}

\subsection{Objetivos}

Siendo conscientes de que la RSC se está convirtiendo en un elemento imprescindible en las empresas, pretendemos conocer cómo la gestionan y cómo la comunican los principales grupos de comunicación a través de la información contenida en sus webs corporativas. Pero, ¿por qué realizar el estudio sobre Internet? Porque desde sus orígenes, este medio se ha convertido en una herramienta clave para la comunicación, ya no solo como soporte publicitario, sino como fuente de información primaria, directa y supervisada por el propio emisor.

Con el tiempo, su uso se fue democratizando y se fue gestando como una plataforma única, con unas características que la diferencian y alejan de los demás medios y soportes convencionales. Su interactividad, inmediatez, hipertextualidad, multimedialidad y la personalización de los servicios la hacen imprescindible (Pareja, 2006: 239-240) al igual que su accesibilidad desde casi cualquier lugar y en cualquier momento.

Todas estas características reinan también en el soporte de referencia de la red, las páginas web. Su usabilidad (facilidad de uso de la página), accesibilidad (facilidad de acceso y manejo de la web y sus contenidos), diseño y contenidos permiten a los usuarios ser parte activa, compartiendo y obteniendo información a través de las redes sociales, foros, comunidades virtuales, blogs, microblogs y demás herramientas. De este modo, la empresa se acerca al público de una forma mucho más familiar, haciendo que los internautas puedan recibir de primera mano información directa a la vez que participan y muestran sus opiniones.

Además, si tenemos en cuenta las cifras publicadas en el resumen del Estudio General de Medios sobre Internet, en los meses de octubre de 2011 a mayo de 2012, estamos ante un medio con un $43.4 \%$ de penetración en la sociedad, el cual, como demuestra el estudio, aumenta gradualmente.

Como aclaran Gallego, García y Rodríguez (2009: 113),

"actualmente en nuestro país las sociedades anónimas cotizadas deben incluir información obligatoria en sus páginas web que se concreta en Información Financiera e Información sobre Gobierno Corporativo, pero además también pueden revelar otro tipo de información que tiene carácter voluntario, siempre respetando el principio de transparencia”.

Por todo ello, consideramos relevante el estudio de la información sobre RSC recogida en los sites corporativos de las empresas de comunicación que conforman la muestra, ya que Internet se ha convertido en un medio esencial para hacer llegar información al público y es un gran portal para mostrar las actividades no lucrativas realizadas. 
Nuestro primer objetivo radica en conocer si los grupos de comunicación informan de las actividades que llevan a cabo en términos de RSC a través de sus webs. En caso afirmativo, se pasará a analizar esta información para dar respuesta a las siguientes preguntas: ¿Cómo definen el conjunto de actividades no lucrativas que realiza la empresa?, ¿aclaran el significado del término RSC?, ¿cuáles son los principales grupos de interés de estas empresas?, ¿existe algún apartado online dedicado exclusivamente a la RSC?, ¿cuáles son los principales temas y actividades no lucrativas que realiza cada grupo?, ¿hay acceso a las memorias o informes en los que se haga referencia a la gestión de la responsabilidad?

Esto nos llevará, por último, a la obtención de las conclusiones pertinentes sobre la aplicación, reconocimiento y creación de valor de la RSC para refutar o no las hipótesis de partida.

\subsection{Metodología}

La investigación, de carácter cualitativa, se desarrolla en torno a tres fases. En primer lugar definiremos el objeto de estudio, revisando en profundidad el concepto RSC y estudiando cuál es su papel en la gestión, en la planificación y en el logro de los objetivos a nivel empresarial.

Una vez comprendido el objeto de estudio iniciaremos una fase de análisis de contenido web centrado en el estudio de los sites corporativos de las empresas que componen la muestra que, recordamos, son PRISA, Planeta, Gestevisión-Telecinco, Vocento, Godó, Intereconomía y Unidad Editorial. Pretendemos con ello dar respuesta a las preguntas planteadas en los objetivos, prestando especial atención a la ya explicada doble responsabilidad de las empresas de comunicación. Posteriormente, estos datos serán interpretados a fin de establecer las conclusiones necesarias para así poder cumplir con los objetivos previamente planteados.

\subsection{Hipótesis}

Al inicio de la presente investigación asumimos que la RSC se ha convertido en uno de los elementos clave que existen en la actualidad para que los organismos de carácter privado se afiancen y adquieran reputación social. Teniendo en cuenta este dato, consideramos que los grupos de comunicación analizados muestran al público las actividades no lucrativas que realizan con el fin de ganarse su confianza y mejorar su percepción social.

Teniendo en cuenta las posibilidades de Internet, partimos de la idea de que las webs de PRISA, Planeta, Gestevisión-Telecinco, Vocento, Godó, Intereconomía y Unidad Editorial utilizan este medio como plataforma comunicativa para dar a conocer las actividades no lucrativas realizadas y cumplir así con el objetivo planteado en la primera de las hipótesis.

Por último, decimos que estas empresas harán una clara distinción de la información sobre RSC en función del público al que se dirigen, prestando especial atención en hacer llegar la actividad realizada a los accionistas. 


\section{Marco teórico}

\subsection{El concepto de responsabilidad social corporativa}

Hoy en día las empresas se conciben como entes que forman parte de una estructura social con la que mantienen una relación constante y con la que se implican a nivel social y/o medioambiental (De la Cuesta, 2006). Esta nueva situación ha llevado a las instituciones a incluir en sus programas un compromiso voluntario de comportamiento responsable que va más allá de la tradición, la legislación o las costumbres. Es lo que se conoce como RSC.

El origen del concepto lo encontramos en los Estados Unidos hacia la segunda mitad del siglo XX, cuando las empresas comenzaron a elaborar los primeros informes de RSC con el fin de mostrar a los públicos externos en qué consistía parte de su actividad (Server y Villalonga, 2005). Las organizaciones tomaron conciencia de que no sólo tenían que velar por sus resultados económicos sino que como entes sociales debían de devolver a la ciudadanía parte de sus beneficios (Marín Calahorro, 2008). En Europa se da el primer paso en 1993 de la mano de Jacques Delors, que intenta implicar a los empresarios en actividades contra la exclusión social (Morrós y Vidal, 2005).

Ya en España, el desarrollo de la RSC se sitúa en el momento de expansión internacional de los años noventa, tal y como indican Albareda y Balaguer (2007) ya que, al traspasar las fronteras, las empresas tuvieron que adaptarse a las políticas internacionales que ya mostraban su preocupación por mantener actividades responsables. En este momento entran también los primeros productos bancarios éticos que promueven el ahorro responsable de las familias, que de alguna forma se veían afectadas por los recientes escándalos financieros. A partir de entonces se hizo más común la preocupación por desarrollar y mantener programas de RSC en las empresas, poniendo especial interés en mantener de una constante comunicación con los grupos de interés o stakeholders, entre los que se encuentran, como indica Ruiz Mora (2010):

- "Propietarios.

- Directivos y/o accionistas.

- Representantes de los trabajadores.

- Trabajadores.

- Autoridades públicas e instituciones.

- Comunidad local.

- Proveedores.

- Consumidores, clientes y/o usuarios.

- Medios de comunicación.

- ONG.

- Competencia"”.

1 De acuerdo con Ruiz Mora (2010), la competencia es un grupo que no se tiene en cuenta como grupo de interés para la empresa. Con frecuencia la conducta responsable de la empresa influirá en el comportamiento de sus competidores, en ocasiones empresas del mismo sector se alían o participan conjuntamente en el desarrollo de acciones o campañas de RSC. 
A pesar de que es un fenómeno relativamente reciente, su importancia la ha convertido en un tema central de debate. Existe una diversidad de definiciones, entre las que se destaca la recogida en el Libro Verde de la Comisión de las Comunidades Europeas (2001) en la que se especifica que es la "integración voluntaria, por parte de las empresas, de las preocupaciones sociales y medioambientales en sus operaciones comerciales y sus relaciones con sus interlocutores". Por su parte, el Consejo Mundial Empresarial para el Desarrollo Sostenible la define como "el compromiso de las empresas de contribuir al desarrollo económico sostenible, trabajando con los empleados, sus familias, la comunidad local y la sociedad en general para mejorar su calidad de vida" (Albareda y Balaguer, 2007: 14-15).

Como indica Orjuela (2011: 145), de las empresas se espera un mayor compromiso tanto con el medio ambiente como con el entorno, lo que le lleva a tener en cuenta que:

- "Ser parte de ese entorno genera responsabilidades a la empresa.

- El entorno merece ser desarrollado por el bien que ese desarrollo significa en sí mismo, aunque ese bien termine beneficiando a la empresa.

- No resulta empresarialmente razonable ni posible esperar que ese entorno o contexto se desarrolle por sí solo, sin la promoción y el apoyo de la empresa, aspectos ambos (la promoción y el apoyo) íntimamente vinculados con la cultura, el sentido y la comunicación".

En definitiva, la RSC se centra en la aplicación voluntaria de la llamada Triple Bottom Line Approach ("triple cuenta de resultados") que incluye actuaciones responsables a tres niveles: económico, social y medioambiental (Marín Calahorro, 2008). Con ello, las empresas generan valor a partir de los activos intangibles puesto que mejoran la percepción social de las empresas y pueden mantener una identidad, mejorar su imagen, legitimarse socialmente y adquirir la reputación corporativa deseada porque, en definitiva, la RSC más que un gasto es una inversión (Moneva, 2007).

\subsection{Identidad corporativa, imagen y reputación como conceptos clave}

A través de la RSC las empresas transmiten una filosofía y cultura que las relaciona con una forma de actuar determinada relacionada, en términos generales, con su preocupación con el entorno y la demostración del buen hacer, transmitiendo una determinada identidad asociada a valores, creencias y formas de actuar concretas y planificadas previamente desde el punto de vista del emisor. En este sentido, todas las actividades llevadas a cabo en el marco de la RSC pueden determinar la forma en la que las organizaciones son percibidas socialmente llegando a afectar incluso a su imagen y, yendo más lejos, a afianzar o a destruir la reputación conseguida a lo largo de los años.

Siguiendo a Orjuela (2011: 144),

"La empresa debe entender que todo lo que hace (y cómo lo hace) construye significados para el entorno, lo cual es muy importante a tener en cuenta, pues esto se puede 
aprovechar para la generación de valores en la sociedad, a través de la comunicación de su propia identidad, bebiendo de la retroalimentación que le puedan generar sus stakeholders".

Llegados a este punto, diferenciaremos tres conceptos que, en ocasiones, se emplean de forma indiscriminada para definir la RSC. Estos conceptos son: "identidad", "imagen" y "reputación". Cuando hablamos de la identidad de la organización, tenemos que tener en cuenta que, como señala Costa (2003),

“etimológicamente, identidad viene de ídem, que significa ‘idéntico', pero idéntico a sí mismo y no a otra cosa. Así, la identidad implica la dialéctica de la diferencia: el ser, o el organismo que es idéntico a sí mismo es, por tanto, diferente de todos los demás”.

En este sentido citaremos a Mínguez (2000) quien, centrándose en el comportamiento de las empresas, indica

"que toda organización realiza diariamente un cierto número de operaciones en el desarrollo de su actividad: fabrica, compra, vende, administra, planifica, contrata, despide... En cada una de estas operaciones la organización lleva a cabo una proyección o una representación de sí misma para los distintos grupos de personas con los que se relaciona".

Es decir, es lo que quiere ser, la forma que tiene la organización de representarse a sí misma ante los públicos y que depende, por lo tanto, de su propia forma de actuar.

Por su parte, la imagen corporativa hace referencia a la percepción o idea mental que los públicos crean en su mente a partir, precisamente, de la percepción de dicha identidad. Como puntualiza Capriotti (2009a: 105) "es un conocimiento espontáneo, fruto de experimentar la presencia de la organización como una persona u objeto más dentro del devenir cotidiano de la vida de un individuo". Vemos por lo tanto como a pesar de que estamos ante dos conceptos que se superponen, estos no tienen que coincidir ya que la identidad que se quiere transmitir puede no estar generando la imagen corporativa deseada en la mente de los públicos.

Cuando hablamos de reputación estamos hablando de un concepto más permanente fruto de "la percepción que los demás tienen, la idea que se forman, el juicio o la valoración que emiten sobre la organización" (Ramos, 2002: 35). Al igual que la imagen, la reputación se genera fuera de la empresa y depende de su buen hacer. La diferencia entre estos dos conceptos reside en que la imagen es más coyuntural mientras que la reputación es fruto del buen hacer empresarial a lo largo del tiempo, lo que dota a la empresa de cierto reconocimiento entre sus públicos, haciéndola más fuerte y generando un gran valor añadido que podrá reforzar a la empresa en situaciones adversas.

Como indica Orjuela (2011: 139),

"la organización comunica a través de lo que hace y hasta de lo que deja de hacer; comunica con sus mensajes planificados, a través de sus marcas, productos y servicios; con su comportamiento legal, fiscal y financiero; con su relación con los proveedores; con la comunidad, a través de los empleados y sus familias; y en general, con todos los públicos con los que deba relacionarse de acuerdo con la naturaleza y entorno del negocio". 
Y, por ello, como concluye Capriotti (2009b),

"la imagen o reputación se establece, fundamentalmente, como una estructura cognitiva de los públicos, como resultado del proceso interno de consumo de la información que realizan los individuos, que dará como resultado la estructura mental de la empresa [...] que originará luego unas valoraciones y unos procesos conductuales determinados".

\subsection{La doble dimensión de la RSC en las empresas de comunicación}

Las empresas de comunicación funcionan como cualquier otra entidad dedicada a la actividad mercantil. Sin embargo, no podemos olvidar que como informadores objetivos de la realidad que nos rodea, estas organizaciones cumplen una función social que cobra especial relevancia dada su doble dimensión en términos de responsabilidad:

- Como empresa: devolviendo a la sociedad parte de sus beneficios e implicándose de forma responsable con su entorno (cf. González, 2007: 45).

- Como organismo transmisor de información: juega un papel muy importante a nivel social ya que los medios y sus soportes son un elemento fundamental a la hora de conformar la opinión pública. En este sentido, encontramos la segunda vía de responsabilidad que tienen que tener las empresas de comunicación: transmitir la información de forma rigurosa, veraz, objetiva y enmarcada dentro de las prácticas éticas. Además, esta función cobra especial relevancia en la actualidad con la influencia que Internet está alcanzando, por lo que "la ética integral de la comunicación es el rasgo que debe definir al nuevo periodismo en una sociedad cada vez más potencialmente abierta a la difusión y recepción de mensajes" (Casasús, 2001: 51).

Como puntualizan Campos, García y Salgueiro (2010),

"resulta evidente que la estructura empresarial y la presión del mercado ejercen una gran influencia sobre la actividad de los medios; a menudo se ven envueltos en tensiones derivadas de exigencias contrapuestas entre sí: las de obtener el mayor beneficio posible como empresas y las de prestar un servicio a la sociedad como medios de comunicación".

\section{Análisis de la RSC a través de las páginas web de los grupos de comunicación}

\subsection{El Grupo PRISA}

Su página electrónica dedica una sección exclusiva sobre RSC, donde se recogen los valores de la empresa, sus líneas de actuación, preocupaciones sociales y medioambientales así como los públicos a los que se dirige. Aunque los proveedores, inversores, accionistas y analistas son sus principales grupos de interés y ofrece una sección exclusiva que ofrece información financiera, los empleados y los medios de comunicación también son un valor importante: dedican la sección "Recursos Humanos" para la gestión interna de los trabajadores actuales y potenciales, así como un espacio llamado "Sala de Prensa", en el que se tiene acceso a la información que éstos podrían requerir: notas de prensa, noticias, entre otros. 
Su línea de actuación responsable gira en torno a dos elementos clave:

Figura 1: líneas de actuación responsable reconocidas en la web de PRISA.

\begin{tabular}{|c|c|}
\hline \multicolumn{1}{|c|}{ Nuestro compromiso } & Nuestras iniciativas \\
\hline Informando con responsabilidad & Periodismo: haciendo escuela \\
\hline Comprometidos con la educación & Fomento de la educación \\
\hline Entretenimiento responsable & Promoción de la cultura y las artes \\
\hline Gestión responsable & Acción Social y Medioambiente \\
\hline
\end{tabular}

Fuente: elaboración propia.

A continuación, procedemos a detallar estos compromisos e iniciativas:

- Informar con responsabilidad y garantizar un entretenimiento responsable. Su objetivo es velar por el interés general, como demuestra su adhesión al Pacto Global de la ONU (http://www.unglobalcompact.org/). Lucha por la existencia de rigor, honestidad, derecho a la información y a la libertad de expresión respetando todas las ideas, culturas y personas. Para ello se ha adaptado a los estándares de ética profesional como el Estatuto de Redacción, el Libro de Estilo o el Defensor del Lector, además de desarrollar códigos éticos como el Código para el Fomento de la Autorregulación sobre Contenidos Televisivos e Infancia o el Código Ético de Conducta Periodística.

- Comprometerse con la educación y fomentar y promocionar de la cultura, las artes y la labor periodística de excelencia, tanto a nivel escolar como universitario y profesional. Ofrece oportunidades de carrera a los estudiantes mediante productos educativos para la formación tanto escolar como ejecutiva, promoviendo la cultura y la lengua española y fomentando la creación literaria, el cine o la música mediante concursos, premios y reconocimientos a nivel individual y empresarial. A través de la Fundación Santillana (http://www.fundacionsantillana.com) vela por la investigación científica y su divulgación, además de realizar actividades dirigidas a estudiantes para el desarrollo de capacidades y aptitudes propias del periodismo (reflexión, actitud crítica, etc.) y elaborar acuerdos de carácter cultural con diferentes instituciones.

- Realizar una gestión responsable a la hora de distribuir la riqueza y crear valor, atendiendo a las demandas y necesidades de los grupos de interés. Para ello desarrollaron una "Guía de Relación con los Proveedores" para garantizar el cumplimiento de todas sus obligaciones, como la protección del medioambiente. Por ejemplo, los soportes El País y 
Cinco Días publican mensualmente los suplementos “Tierra” y "Entorno” respectivamente.

- Participar en campañas solidarias, cediendo espacios publicitarios a organizaciones no lucrativas u ONG, así como llegar a acuerdos de patrocinio "convirtiéndose en altavoces de las campañas solidarias y defensa de los Derechos Humanos".

\subsection{Grupo Planeta}

Su filosofía corporativa se fundamenta sobre cuatro ideas básicas:

- Las personas.

- La ética.

- La calidad.

- La excelencia en el trabajo.

Su actividad no lucrativa y filantrópica se realiza a través de la fundación José Manuel Lara (http://www.fundacionjmlara.es/). Esta fundación se centra en promocionar la cultura hispana, en especial la andaluza, en fomentar la lectura, organizar eventos culturales y conceder premios de literatura, de investigación en humanidades y de novela.

Además, ofrece información a los medios de comunicación a través de "notas de prensa" y reserva un apartado de acceso restringido para los proveedores.

\subsection{Grupo Gestevisión-Telecinco}

La información contenida en la página web oficial del grupo Mediaset España (http:// www.telecinco.es/inversores/es/) se dirige, en especial, al grupo de interés conformado por accionistas e inversores. El site cuenta con un buscador interno propio, actualizaciones constantes de la cotización en bolsa de sus acciones y botones de acceso directo a los resultados de la Junta General de Accionistas 2011, al Informe Anual, a la última presentación de resultados, a hechos relevantes y a las últimas noticias del grupo, satisfaciendo las necesidades de accionistas y medios de comunicación.

La RSC se recoge en la sección "Responsabilidad Corporativa", donde se puede acceder a la información al respecto de cada año desde 2005. La información contenida en su último informe de RSC podemos clasificarla de la forma siguiente:

- Consolidarse como un referente en gestión ética en el sector de la información y el entretenimiento. Para ello participa en el desarrollo de políticas públicas, siendo miembro del Consejo Asesor para las Telecomunicaciones y la Sociedad de la Información del Ministerio de Industria, Turismo y Comercio; participa y acepta los estándares establecidos por la Global Reporting Initiative desde 2009 y apoya el Pacto Mundial de Naciones Unidas (http://www.unglobalcompact.org/) centrado en la implantación y protección de los derechos humanos, laborales, medioambientales y anticorrupción. Además, basa su Sistema de Gestión de Riesgos Corporativos en la transparencia, eficacia, eficiencia y fiabilidad de sus operaciones y activos. 
- Política medioambiental.Participa en la iniciativa de Naciones Unidas Carbon Disclosure Project (https://www.cdproject.net), centrada en aportar soluciones al cambio climático, reduciendo sus emisiones de gases efecto invernadero. Forma parte del FTSE4Good Ibex, que "reconoce los niveles de gestión medioambiental, de derechos humanos y política laboral"; está en el ranking Global 100 Sustainable Performance Leader como empresa sostenible y en Triodos Bank como empresa "elegible para invertir de forma socialmente responsable".

- Presta especial atención a los accionistas, aunque son conscientes de la importancia de todos los stakeholders.

- Control de contenidos bajo dos perspectivas de actuación. Por un lado, la emisión de contenidos "de elevado impacto social a través de campañas específicas y programas cuya finalidad fuera el fomento de valores sociales y sostenibles" y, por otro, "dar cobertura informativa a asuntos de interés social [...] asegurando el cumplimiento de los estándares éticos suscritos", velando por la accesibilidad y cumpliendo el Código de Autorregulación de Contenidos Televisivos e Infancia.

\subsection{Grupo Vocento}

Ejerce sus acciones de RSC a través de la Fundación que lleva su mismo nombre, a la que se accede a partir de la sección "Acción Cultural".

Sus actividades no lucrativas se centran en la promoción de la cultura, la educación, los valores cívicos y el talento. Para ello promueve concursos, entrega premios, organiza eventos, colabora con instituciones educativas y publica la revista digital El Noticiero de las Ideas.

Dentro de la sección “Accionistas e Inversores” se puede acceder a información financiera del grupo, a sus resultados mensuales y a su Memoria Anual (http://www.vocento.com/ info_anual.php), que dedica un apartado a la RSC. En ella destaca la importante función social de los medios de comunicación, el respeto y defensa de los Derechos Humanos, la infancia, el honor, la intimidad, la igualdad de género y el combate a la corrupción, de ahí su renovación con su compromiso con el Pacto Mundial a favor de una sociedad sostenible.

A través de "Sala de Prensa", los medios tienen acceso a las notas de prensa y a las noticias publicadas y relacionadas con el grupo.

\subsection{Grupo Godó}

No hace referencia en ningún momento a actividades de RSC, sino que habla de mecenazgo ramificado en cuatro líneas de actuación:

- La cultura: a través de la Fundación Conde de Barcelona fomenta las ciencias y la cultura reconociendo el trabajo realizado en comunicación, así como la edición de libros de interés histórico. 
- La educación: patrocina eventos culturales y educativos, además de colaborar con instituciones universitarias catalanas.

- El deporte: impulsa la celebración de eventos deportivos y colabora en la organización otras actividades como la Gran Gala del Deporte, el Trofeu Campions, la carrera Jean Bouin o el Prix Pyrenées.

- La economía: participa en la organización de salón Barcelona Meeting Pont o en las sesiones de Matins Esade.

Los grupos de interés en los que se centra el contenido de su web son los medios de comunicación, los recursos humanos y la sociedad en general a través de las actividades no lucrativas.

\subsection{Grupo Intereconomía}

El grupo ofrece información acerca de su actividad no lucrativa a través de la sección "Otras Áreas", concretamente a través de "Intereconomía Proyectos" (www.intereconomia proyectos.com/) y la Fundación Intereconomía (http://www.intereconomiaproyectos.com/ fundacion.html).

Su objetivo, tal y como puntualizan en el site, es “contribuir y fomentar la difusión de valores fundamentales" para ayudar a los más desfavorecidos mediante premios y colaboraciones con otras empresas o instituciones, recogiendo donativos o realizando acciones conjuntas de mecenazgo. Los principales temas de actuación responderían a su "compromiso de respeto efectivo en los derechos y libertades fundamentales en el marco de una sociedad libre, justa, veraz, solidaria y democrática".

Su plan de acción se centra en los siguientes puntos:

Figura 2: líneas de actuación del Grupo Intereconomía.

\begin{tabular}{|c|c|}
\hline Nuestro compromiso & Nuestras iniciativas \\
\hline Familia y reconciliación & Medio ambiente \\
\hline Salud y calidad de vida & Sostenibilidad \\
\hline Mujer & Diversidad \\
\hline Responsabilidad Social Corporativa & Cooperación \\
\hline Inmigración & Prevención de riesgos laborales \\
\hline
\end{tabular}




\begin{tabular}{|c|c|}
\hline Violencia de género & Foros tecnológicos \\
\hline Team Building & Networking \\
\hline Jornadas con expertos & Congresos \\
\hline Premios & Guías y estudios \\
\hline I+D & Adopción y acogimiento familiar en la infancia \\
\hline
\end{tabular}

Fuente: elaboración propia.

Además, fomenta la educación a través de la Escuela de Finanzas y de la Cultura, recuperando obras de la literatura universal a través de Bibliotheca Homo Legens.

\subsection{Unidad Editorial}

El grupo se define como "referencia, a través de sus productos, en calidad, rigor, independencia en el ejercicio del mejor periodismo en la Prensa española". La información sobre la RSC del grupo Unidad Editorial se localiza bajo los epígrafes "RSC Media Group" y "Responsabilidad Social” de la sección “información corporativa”. En el primero se define la estrategia del grupo en materia medioambiental, alineando la política del departamento de Facility Management con la legislación, comunicándola a los implicados, velando por el desarrollo sostenible e intentando mejorar a cada paso. Sus áreas de actuación son cuatro y se puede consultar en la auditoría de medio ambiente del grupo (http://www. unidadeditorial.com/descargas/medioambiente-2010.pdf):

- Gestión energética: promoviendo el consumo responsable de energía y recursos.

- Residuos: centrado en reducir la basura generada en las instalaciones del grupo.

- Compras: reducir la contaminación mediante la contratación de distribuidores que utilizan medios de transporte que emiten menos partículas perjudiciales.

- Industrial: a través de ECOEMBES, asociación sin ánimo de lucro a la que está adherida, recogen y recuperan residuos de envases para su tratamiento y reciclado.

En "Responsabilidad Social" se recoge su código ético, basado especialmente en:

- Generar valor empresarial para satisfacer a los accionistas, manteniendo relaciones constantes con el mercado financiero para que estos se vinculen con actividades sociales.

- Fomentar el crecimiento cultural del país ofreciendo información completa, íntegra y pluralista evitando las discriminaciones: actúan bajo los criterios de transparencia, claridad, 
eficacia y eficiencia, con el fin de cumplir con las expectativas de todos los grupos de interés.

- Velar por su público interno, transmitiendo la importancia del trabajo en grupo en un entorno seguro y adecuado para mejorar los resultados. Reconoce y valoriza a cada integrante del mismo teniendo en cuenta sus opiniones y haciéndolos partícipes de las decisiones del grupo bajo las cláusulas legales que se estipulan, fomentando la competitividad como elemento generador de valor en un mercado exigente y amplio, manteniendo una relación leal con la competencia.

- Clientes y proveedores tienen la posibilidad de acceder a un site privado para comprobar sus facturas.

Además, el grupo apoya iniciativas de instituciones que tienen que ver con la promoción de la cultura, educación, valores cívicos, deportivos, ambientales, etc. siendo en ocasiones precursor de campañas de sensibilización.

\section{Resultados}

Tras el análisis realizado comprobamos como, tal y como afirma Viñarás (2010),

"las organizaciones están adoptando un papel que tradicionalmente correspondía a las instituciones públicas, el de promover y desarrollar programas sociales, ya sea sobre medioambiente, acción social o la promoción del arte y la cultura; asumiendo responsabilidades que hasta ahora no les eran aplicables, y que en pocos años se han convertido en un rasgo diferencial de su personalidad".

Vemos que los grupos de comunicación estudiados muestran una vinculación directa con actividades socialmente responsables sin ánimo de lucro: compromiso social, fomento de la cultura, apoyo a los deportes o el respeto y cuidado del medio ambiente. Aunque ninguno define la RSC, PRISA, Gestevisión-Telecinco y Unidad Editorial utilizan dicho término para definir estas actividades. Por su parte, Vocento habla de "acción cultural", Godó de "mecenazgo" e Intereconomía realiza sus acciones a través de "Intereconomía Proyectos" y de su fundación, al igual que Planeta.

A pesar de que con el tiempo las empresas han tomado conciencia de que hay grupos de interés más allá de los accionistas, estos siguen siendo el principal sector al que se dirigen grupos como PRISA, Gestevisión-Telecinco y Vocento, que ofrecen información relevante sobre cotización, capital social, dividendos, resultados, entre otros. Sin embargo, cada vez se tienen más en cuenta los siguientes aspectos:

- Recursos Humanos: cobra especial relevancia para PRISA, Vocento y Godó, que les dedican una sección en exclusiva. Gestevisión-Telecinco los incluye en su informe anual del año 2010; en Intereconomía la prevención de riesgos laborales es uno de sus ejes de actuación y Unidad Editorial reconoce la importancia de valorizar a cada miembro de la empresa para mejorar sus resultados.

- Medios de comunicación: son conscientes de su importancia para la transmisión de una 
determinada identidad corporativa entre los públicos a los que se dirige, por ello todos ofrecen información acerca de las noticias en las que son protagonistas.

- Audiencia: los siete grupos velan por su protección y respeto.

- Proveedores: el grupo PRISA, Planeta y Unidad Editorial reservan un espacio de acceso restringido para ellos, mientras que Gestevisión-Telecinco hace eco de su importancia en el informe anual.

El respeto al medio ambiente, uno de los Triple Bottom Line Approach, se ha convertido en un elemento clave en las empresas que toman "medidas verdes" para mostrar su compromiso con el desarrollo sostenible y la preservación de los recursos naturales. En este caso es el grupo Gestevisión-Telecinco el que más información y preocupación muestra al respecto, adhiriéndose a normativas internacionales y a programas medioambientales.

Por otro lado, vemos que en los grupos de comunicación es muy común la creación de fundaciones que, bajo el mismo nombre que la empresa, participan en la esfera pública realizando una labor social no lucrativa mediante la financiación de actividades de interés general con dinero privado. Como indica Alfaro (2005: 510),

"frente a las acciones de patrocinio de las organizaciones, la creación o apoyo a fundaciones permite a las organizaciones reconstruir la confianza en sus públicos concentrando su participación en actividades de interés general destinadas a un colectivo identificado, les permite desvincularlas del resto de sus actividades y con ello, ganar en credibilidad y rentabilidad".

Dentro de la RSC, estas actividades no lucrativas buscan el equilibrio entre la obtención de beneficios y la reinversión de estos en la sociedad para lograr transmitir la identidad corporativa deseada y generar una reputación que legitime socialmente a la empresa aumentando el valor de sus marcas.

\section{Conclusiones}

Tras el análisis realizado, podemos concluir que los grupos de comunicación son conscientes de la necesidad e importancia de dar a conocer, a través de sus páginas web, su filosofía, sus valores, sus principios corporativos, políticas y actuaciones de carácter filantrópico con el fin de mejorar su imagen y reputación, algo que se puede traducir en beneficios a corto, medio o largo plazo.

Sin embargo no podemos decir lo mismo si hablamos del término RSC propiamente dicho, ya que ninguna de las empresas analizadas explica o aclara el concepto en su site corporativo. Así como el grupo Gestevisión-Telecinco facilita el acceso a un informe o memoria de RSC anual y la web de PRISA podría considerarse como tal dada la cantidad y claridad de la información ofrecida al respecto, los cinco grupos restantes no profundizan en el tema a pesar de las posibilidades de Internet para hacerlo. Existe una mayor atención a actividades de mecenazgo relacionadas con la promoción de la cultura a través de fundaciones y otros 
organismos no lucrativos, por lo que concluimos que los grupos de comunicación no están sacando el máximo partido a las posibilidades que Internet les ofrece para hacer llegar al público sus actuaciones responsables.

Sin embargo, como webs de empresas de comunicación que son y cumpliendo la doble RSC de la que hablábamos al inicio de la investigación, toman consciencia de su función como generadores de opinión pública y transmisores de la realidad de su entorno, asumiendo y defendiendo de forma directa su función de velar por el periodismo objetivo, veraz y riguroso, adhiriéndose en muchos casos a códigos éticos nacionales o internacionales o incluso creando los suyos propios.

Respecto a la última de las hipótesis de partida establecida al inicio del estudio, vemos como, aunque son conscientes de la importancia adquirida por todos sus stakeholders, los accionistas siguen siendo el colectivo al que, en general, se le dedica más espacio y se le ofrece más información, ya que la RSC no deja de ser una estrategia empresarial para general valor en los intangibles. Aunque hablamos de empresas de comunicación con medios y soportes de carácter privado (que sobreviven, en su mayoría, gracias a la inversión publicitaria realizada por los anunciantes) el colectivo más importante para el grupo son los accionistas, de cuya inversión depende en gran parte, la viabilidad de la organización.

\section{Fuentes consultadas}

- Albareda Vivó L. y Balaguer Franch M. (2007). "La responsabilidad social de la empresa y los resultados financieros". Responsabilidad Social Corporativa, pp. 11-26.

- Alfaro Lara, M. (2005). "La comunicación de la responsabilidad social corporativa: la fundación de empresa como elemento estratégico",pp. 509-538. En Castillo Esparcia,A. (coord.). Comunicación organizacional. Teorías y estudios. Málaga: Clave Aynadamar.

- Campos Freire, F.; García, F. y Salgueiro Santiso, M. (2010). "Las empresas de comunicación ante la Responsabilidad Social Corporativa". Ponencia presentada en el Congreso Internacional AE-IC Málaga Comunicación y Desarrollo en la era digital. Málaga. Extraída el 30/V/2012 desde http://www.aeic2010malaga.org/esp/secciones_ det.asp?id_seccio=7\&id_slot=5\&id_seccion $=7$

- Capriotti Peri, P.

_(2009a). Branding Corporativo. Fundamentos para la gestión estratégica de la Identidad Corporativa. Santiago de Chile: Colección de Libros de Empresa.

_(2009b). "De la imagen a la reputación. Análisis de similitudes y diferencias”. Razón y Palabra, núm. 70. Extraída el 29/V/2012 desde http://www.razonypalabra.org.mx/ Articulo\%2013\%20CAPRIOTTI\% 20Imagen\%20y\%20ReputacionR\&P.pdf

- Casasús Guri, J. (2001). "Perspectiva ética del periodismo electrónico”. Estudios sobre el mensaje periodístico, núm. 7, pp. 49-55. Extraída el 20/V/2012 desde http://dialnet. unirioja.es/ servlet/articulo?codigo $=776435$ 
- Costa, J. (2003). "Creación de la imagen corporativa. El paradigma del siglo XXI". Razón y palabra,núm. 34. Extraída el 20/V/2012 desde http://www.www.razonypalabra. org.mx/anteriores/n34/jcosta.html

- De la Cuesta, M. (2006). "Responsabilidad social del sector bancario. Su contribución a un desarrollo más sostenible”. Papeles de la Economía Española, núm. 108, pp. 173-190.

- Estudio General de Medios (2012). "Resumen General de resultados EGM. Octubre de 2011 a Mayo de 2012”. Extraída el 10/VII/2012 desde http://www.aimc.es/-DatosEGM-Resumen-General-.html

- Fernández García, R.

_(2011). La dimensión económica del desarrollo sostenible. Alicante: Club Universitario.

_(2009). Responsabilidad Social Corporativa. Alicante: Club Universitario.

- Freeman, R. (1984). Strategic Management. A Stakeholder Approach. Maryland: Pitman.

- Gallego Álvarez, I.; García Sánchez, I. y Rodríguez Domínguez, L. (2009). "La eficacia del gobierno corporativo y la divulgación de información en Internet". Investigaciones Europeas de Dirección y Economía de la Empresa, vol. 15, pp. 109135. Extraída el 20/V/2012 desde http://redalyc.uaemex.mx/ src/inicio/ArtPdfRed. jsp?iCve $=274120370007$

- González, M. (2007). "RSE y Medios de Comunicación”, pp. 45-61. En Galindo García, A. y Fernández Olit, B. (coords.). Responsabilidad Social Corporativa y Medios de Comunicación Social. Salamanca: Universidad Pontificia de Salamanca.

- Manfredi, J. (2006). "Responsabilidad Social Corporativa de las Empresas de Televisión". Ponencia presentada en la conferencia La ética y el derecho en la producción y el consumo del entretenimiento de la Fundación COSO de la Comunidad Valenciana para el Desarrollo de la Comunicación y la Sociedad. Extraída el 20/V/2012 desde http://dialnet.unirioja.es/servlet/articulo?codigo=2341199

- Marín Calahorro, F. (2008). Responsabilidad social corporativa y comunicación. Madrid: Fragua.

- Míguez González, M.I. (2007). “Análisis del uso de los conceptos de público, stakeholder y constituent en el marco teórico de las Relaciones Públicas”. Zer, Revista de Estudios de Comunicación, núm. 23, pp. 183-197. Extraída el 20/V/2012 desde http://www.ehu.es/zer/zer23/ZER23_miguezgzalez.pdf

- Minguez, N. (2000). "Un Marco Conceptual para la Imagen Corporativa”. Zer Revista de Estudios de Comunicación, núm. 8, pp. 303-321. Extraída el 18/V/2012 desde http://www.ehu.es/zer/es/hemeroteca/ articulo/un-marco-conceptual-para-la-imagencorporativa/115

- Moneva, J. (2007). “¿Es la Responsabilidad Social Corporativa rentable para la 